\title{
In Vivo Assessment of Burn Depth and Wound Healing Using a Handheld Terahertz Hyperspectral Scanner
}

\section{Omar Osman \\ Stony Brook University \\ Zachery Harris \\ Stony Brook University \\ Juin Zhou \\ Stony Brook University \\ Mahmoud Khani \\ Stony Brook University \\ Adam Singer \\ Stony Brook Medicine}

M. Arbab ( $\square$ hassan.arbab@stonybrook.edu )

Stony Brook University

\section{Research Article}

Keywords: Terahertz Time-Domain Spectroscopy, skin tissue hyperspectral imaging, handheld THz-TDS scanner, burn imaging, partial-thickness burn characterization, histology.

Posted Date: January 5th, 2021

DOl: https://doi.org/10.21203/rs.3.rs-138599/v1

License: (9) This work is licensed under a Creative Commons Attribution 4.0 International License.

Read Full License 


\title{
In vivo assessment of burn depth and wound healing using a handheld terahertz hyperspectral scanner
}

\author{
Omar B. Osman, $\mathrm{MS}^{1}$, Zachery B. Harris, $\mathrm{BS}^{1}$, Juin-Wan Zhou, $\mathrm{MS}^{1}$, Mahmoud E. Khani, MS ${ }^{1}$, \\ Adam J. Singer, $\mathrm{MD}^{2}$, M. Hassan Arbab, $\mathrm{PhD}^{1}$ \\ ${ }^{1}$ State University of New York at Stony Brook, THz Biophotonics Laboratory, Department of Biomedical \\ Engineering, 101 Nicolls Rd. Stony Brook, NY, USA 11794 \\ ${ }^{2}$ Renaissance School of Medicine at Stony Brook University, Department of Emergency Medicine, 101 Nicolls Rd. \\ Stony Brook, NY, USA 11794
}

Omar B. Osman:

- omar.osman@stonybrook.edu;

- https://orcid.org/0000-0002-0943-6703

Juin-Wan Zhou:

- juin-wan.zhou@stonybrook.edu

- https://orcid.org/0000-0002-7457-2912

Zachery B. Harris:

- zachery.harris@stonybrook.edu

- https://orcid.org/0000-0002-6557-6225

Mahmoud E. Khani:

- mahmoud.ebrahimkhani@stonybrook.edu

- https://orcid.org/0000-0002-3058-6247

Adam J. Singer:

- Adam.Singer@stonybrookmedicine.edu;

- https://orcid.org/0000-0003-4694-6152

M. Hassaan Arbab:

- hassan.arbab@stonybrook.edu;

- https://orcid.org/0000-0002-6565-3358

All work was done in Stony Brook, NY, 11790, USA.

Correspondence: M. Hassan Arbab, PhD

- Phone: 1 (631) 6321050.

- Address: 101 Nicolls Rd. Stony Brook, NY, USA 11794.

- Email: hassan.arbab@stonybrook.edu

Abbreviations used: THz: Terahertz, THz-TDS: terahertz time-domain spectroscopy, TD: time-domain, ANSI: American National Standards Institute, THz-TDSI, terahertz time-domain spectral imaging, CW: continuous wave, ROI: regions of interest, SA: spectral amplitude, SS spectral slope, BW: bandwidth, IM: intramuscular, HDPE: high density polyethylene, H\&E: hematoxylin and eosin, PCA: photoconductive antenna, 


\section{ABSTRACT}

The accuracy of clinical assessment in partial-thickness burn injuries has remained as low as 50$75 \%$. Depending on the depth and environmental factors in the wound, such as reactive oxygen species, inflammation, and autophagy, partial-thickness burns can heal spontaneously or require surgical intervention. In this study, we demonstrate that Terahertz Time-Domain Spectral Imaging (THz-TDSI) is a promising tool for in vivo quantitative assessment of burn injuries. We used a novel handheld THz-TDSI scanner to characterize burn injuries in a porcine scald model with histopathological control. Prior work used THz reflectivity (representation of tissue hydration) as the only source of signal contrast. However, we used the spectral amplitude and the spectral slope of the terahertz electric field to distinguish the different severities of burns, suggesting that the energy loss due to electromagnetic scattering from skin constituents serves an additional metric to quantitatively assess burn injuries. Statistical analysis $(n=40)$ indicates that THz-TDSI can accurately differentiate between partial-thickness and full-thickness burn injuries (1-way ANOVA, $p<0.05$ ) and monitor the healing process of partial thickness burns. THz-TDSI has the potential to improve burn care outcomes by helping surgeons to make objective decisions for early excision.

Keywords: Terahertz Time-Domain Spectroscopy, skin tissue hyperspectral imaging, handheld THz-TDS scanner, burn imaging, partial-thickness burn characterization, histology.

\section{INTRODUCTION}

Acute burn injuries accounted for approximately 489,000 emergency department visits in $2017^{1}$. At triage, burn injuries are assessed by a clinical evaluation, which is a subjective method that depends on visual and tactile inspection of the injury. A physician will determine the course of treatment based on the perceived depth of thermal damage and the total body surface area that has 
sustained thermal injury. Superficial burns affect the epidermis and will heal spontaneously by reepithelialization. Full-thickness burns affect the entire depth of the dermis, can result in hypertrophic or contracted scarring, and will require surgical intervention (i.e. excision and grafting) ${ }^{2}$. Partial-thickness burns, which affect the epidermis and papillary region, are challenging to assess because they may heal spontaneously or progress into deeper thickness burns. Additionally, wound conversion in partial-thickness burns depends on multiple factors, including perfusion ${ }^{3}$, reactive oxygen species ${ }^{4}$, and mechanisms such as autophagy and inflammation ${ }^{4}$. Clinical evaluation for determining whether a partial-thickness burn injury will progress into a full-thickness burn has been shown to have an accuracy as low as $50-75 \%{ }^{5}$. This low-accuracy rate often results in delaying diagnosis until the burn declares its nature. On the other hand, early excision and grafting of full thickness burns results in overall better patient outcomes ${ }^{6,7}$. Therefore, early and accurate determination of partial-thickness burn injuries can reduce the recovery time and financial burdens that are experienced by burn patients.

Terahertz Time-Domain Spectroscopy (THz-TDS) is a coherent broadband spectral imaging technique, where a pulsed terahertz $(\mathrm{THz})$ electric field $(0.1-10 \mathrm{THz})$ is reflected from a sample and measured in the time-domain (TD) with sub-picosecond resolution. $\mathrm{THz}$ radiation has piqued the interest of biomedical researchers because THz-TDS techniques are highly sensitive to the water content of tissue. This is due to the broad absorption of water at $\mathrm{THz}$ frequencies that comes from collective intermolecular motions of bound and free water ${ }^{8}$. Additionally, THz-TDS techniques are coherently detected and are less prone to electromagnetic scattering (compared to smaller wavelengths $)^{9,10}$. Many fingerprint spectral resonances of biomolecules in polycrystalline form also exist in $\mathrm{THz}$ wavelengths ${ }^{9,10}$. These features make THz-TDS a promising imaging modality for diagnosis of burn injuries ${ }^{11,12}$. THz waves are non-ionizing and safe for use in humans 
and animals. Previous safety studies determined that there are no negative effects in vivo and the only thermal effects in vitro are a result of extremely high power and long exposure duration of THz radiation ${ }^{13}$. The American National Standards Institute (ANSI) provides laser safety standards for industrial, military, medical and other applications and their Maximum Exposure Limit of $1 \mathrm{~mm}$ wave radiation (> $0.3 \mathrm{THz}$ frequency, or $>1.2 \mathrm{meV}$ photon energy) is $0.1 \mathrm{~W} / \mathrm{cm}^{2}$ 14. The THz Time-Domain Spectral Imaging (THz-TDSI) scanner introduced in this study is well within this safety standard by using a pulsed system of $<0.37 \mathrm{~mW} / \mathrm{cm}^{2}$ for 0.35 second scan time per pixel.

Several research groups have approached using THz imaging to measure burn injuries, both ex vivo ${ }^{15}$ and in vivo ${ }^{11,12,16}$. An ex vivo THz study of burn wounds on porcine skin samples suggested that the primary contrast mechanism was the water content because there was a reduced reflectivity at the burn site ${ }^{15}$. However, upon the infliction of a burn injury, physiological events, such as increased capillary permeability, increased hydrostatic pressure, and edema, occur and increase the water content ${ }^{17}$. Later $\mathrm{THz}$ studies, using in vivo rat models, showed that the $\mathrm{THz}$ spectra of burns had increased reflectivity 72 hours postburn, which was consistent with overall water content increase due to edema and correlated with the density of structures within the skin ${ }^{11,12,16,18,19}$. Moreover, Suen et al. showed a practical benefit of using $\mathrm{THz}$ radiation over infrared wavelengths because of its ability for transmission and propagation through typical burn wound topical treatments and wound dressings with little attenuation ${ }^{20}$, suggesting that $\mathrm{THz}$ radiation has the potential to monitor burn injuries throughout the healing process with minimal discomfort to the patient.

Another practical consideration for the utility of the THz-TDS modality is its form factor. Most THz-TDS systems require raster scanning a sample around a fixed optical focus, which will not 
be suitable outside research laboratories or small animal studies. To address this issue, a few research groups have developed compact and handheld $\mathrm{THz}$ devices. As an example, a singlepoint spectroscopic handheld device was developed for single-pixel measurement applications ${ }^{21}$, which was also used for measurement dielectric response of human skin ${ }^{22}$. A handheld single-line scanner was designed for imaging malignant breast tumors, however, it was restricted to scanning along a single dimension ${ }^{23}$, with a $2 \mathrm{~mm}$ by $15 \mathrm{~mm}$ field-of-view (FOV). In contrast to these instruments, we have designed and built a handheld, fiber-coupled, two-dimensional THz-TDSI scanner with a maximum FOV of up to $43 \times 27 \mathrm{~mm}$, and have tested its utility in the first in vivo porcine burn study using $\mathrm{THz}$ radiation ${ }^{24,25}$.

In this work, we will present an acute in vivo porcine scald study model, established to demonstrate the accuracy of the THz-TDSI modality to assess burn injuries. While most previous in vivo $\mathrm{THz}$ studies required a challenging alignment setup attached to a cumbersome optical table, we developed the first handheld, alignment-free, and easily deployable THz-TDSI scanner for diagnostic mapping of burn severity in large mammals. We will show that our THz-TDSI device could easily demarcate burned tissue based on the integration of the deconvolved spectral amplitude between the 0.2 to $0.8 \mathrm{THz}$ band, which was within the bandwidth (BW) of our scanner. Also, we will show that using the dual-band hyperspectral slope of the deconvolved $\mathrm{THz}$ amplitude, we can differentiate scald burns of different depths of dermal injury against histological assessments as the gold standard $(p<0.05)$ and monitor their progression.

\section{RESULTS}

\section{$\underline{\text { Hyperspectral analysis }}$}

Visual images, $\mathrm{THz}$ images, and the deconvolved reflectivity, $R(f)$, from the corresponding regions of interest (ROIs) for burn and perilesional skin are shown in Fig. 1. Due to the size of the 
wounds $\left(20 \times 20 \mathrm{~mm}^{2}\right)$ and the smaller FOV setting $\left(12 \times 19 \mathrm{~mm}^{2}\right)$, we collected two THz images to include the burn and perilesional skin. THz images were created by integrating $R(f)$ between the frequency bin with the largest amplitude, $f_{R_{\max }}$, and nearest bin where the reflectivity, before deconvolution, was below $-10.5 \mathrm{~dB}$, defined as $f_{-10.5 d B}$. The integrated reflectivity was then normalized by the number of frequency bins, $N_{\text {bins }}$ to calculate the spectral amplitude, $S A$, given by,

where

$$
S A=\frac{1}{N_{\text {bins }}} \int_{f_{\text {lower }}}^{f_{\text {upper }}} R(f) d f
$$

$$
f_{\text {upper }}=f_{R_{\max }}+f_{-10.5 d B}
$$

and

$$
f_{\text {lower }}=f_{R_{\max }}-f_{-10.5 d B} \text {. }
$$


(a)

(d)

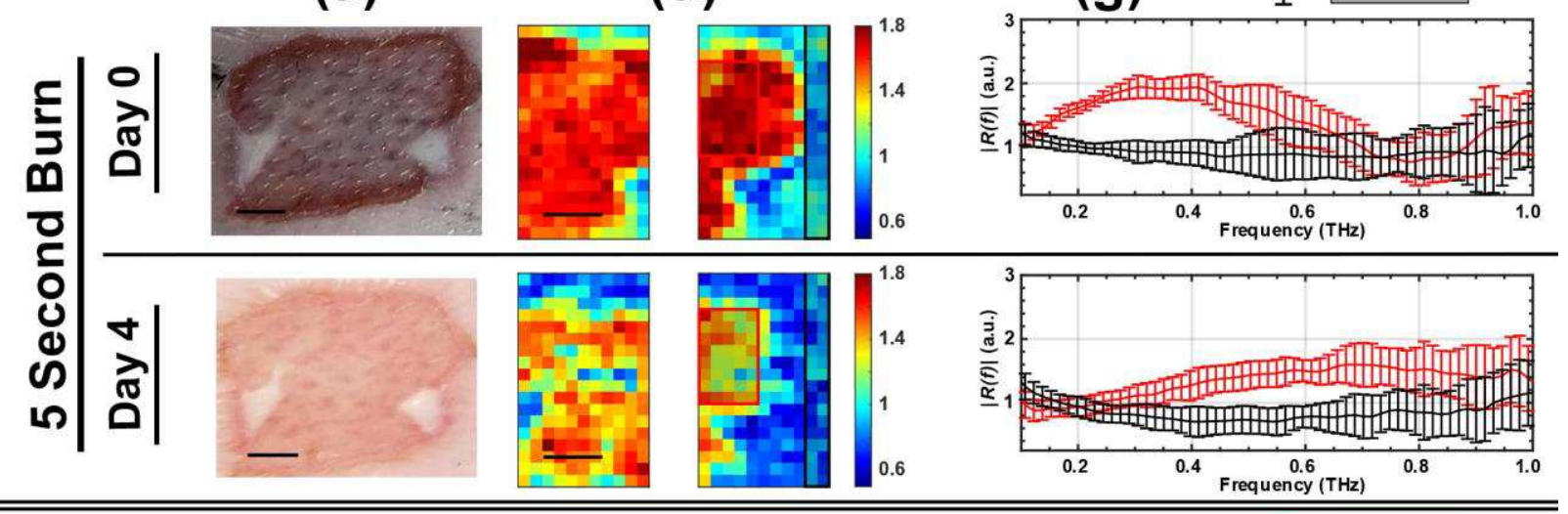

(b) (e)

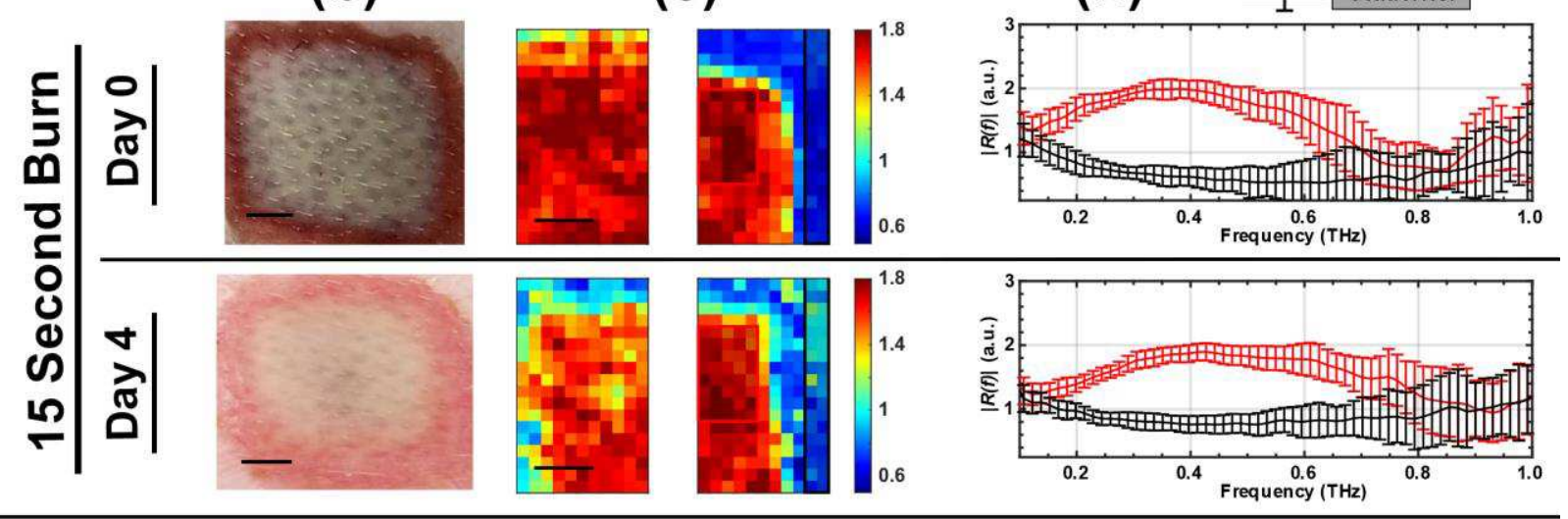

(c)

(f)

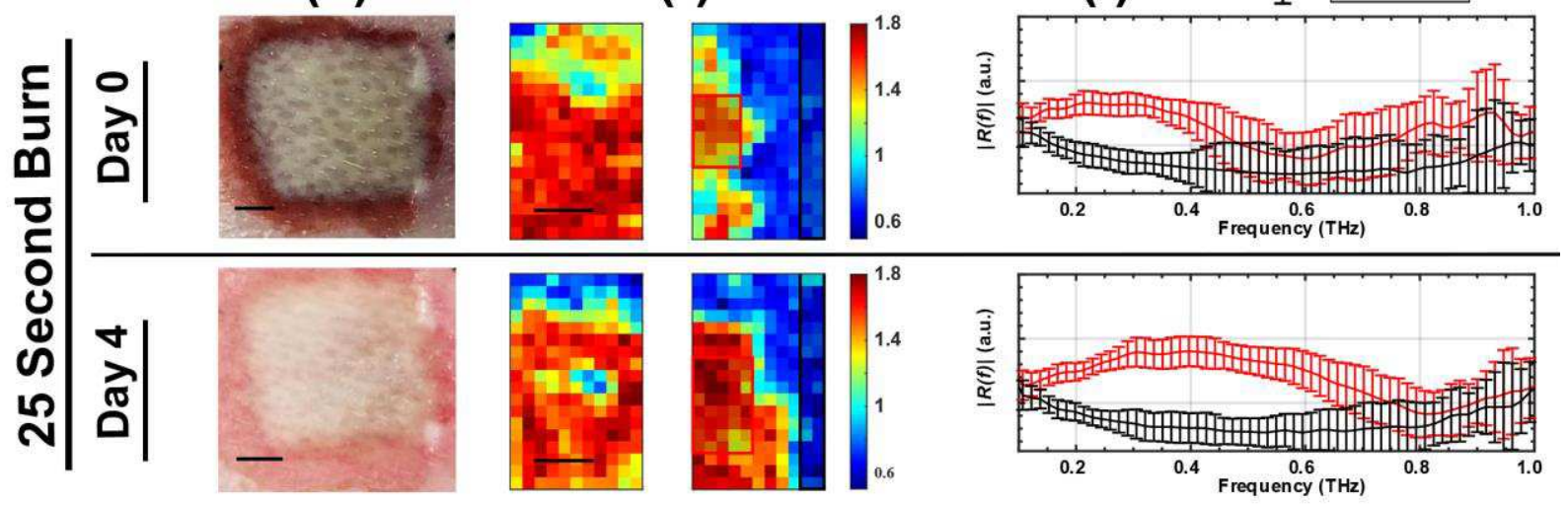

Fig. 1. Results from THz imaging. Visual images (a-b), THz images (c-d), where the quantity shown in the colorbar is $S A$, and representative THz reflectivity of burned (red box) and normal (black box, control) skin within the defined ROI (e-f) (mean +/- SD) are shown for each burn condition on Day 0 and Day 4. There are two side-by-side THz images shown for each $20 \times 20 \mathrm{~mm}^{2}$ burn and the surrounding normal skin region, because the FOV of the THz scanner was limited to $12 \times 19 \mathrm{~mm}^{2}$. (Scale bar $=5 \mathrm{~mm}$ ). 


\section{$\underline{\text { Statistical analysis }}$}

The $S A$ parameter alone did not show clear differences between burns severities when compared to histological assessment. Therefore, we calculated the spectral slope, $S S$, of $R(f)$ by computing the dual-band upper and lower spectral slopes of the linear fit between the frequency bins, $f_{\text {lower }}$ to $f_{R_{\max }}$ and $f_{R_{\max }}$ to $f_{\text {upper }}$, in each pixel of the burned ROI. Our previous work using in vivo rodent ${ }^{12}$ and porcine ${ }^{19}$ models revealed that the $\mathrm{THz}$ spectral slopes contain valuable hyperspectral information. An example of the calculation of $S S$ for a single pixel is shown in Fig. 2(a). Because $S A$ is calculated as a sum across a frequency range, this spectroscopic parameter is inherently less sensitive to frequency dependent behavior. $S S$, however, allows us to quantify frequency dependent changes in each ROI. One example of frequency dependent behavior is electromagnetic scattering, which is wavelength and particle size dependent ${ }^{26}$ and is likely the source of signal contrast in $S S$. As a result, the differences in $S S$ between burn depths suggests that physiological and structural changes in the skin constituents postburn can serve as a source of imaging signal contrast because $\mathrm{THz}$ wavelengths ( $3 \mathrm{~mm}$ to $10 \mu \mathrm{m}$ ) are similar in size to some of these adnexal structures.

(a)

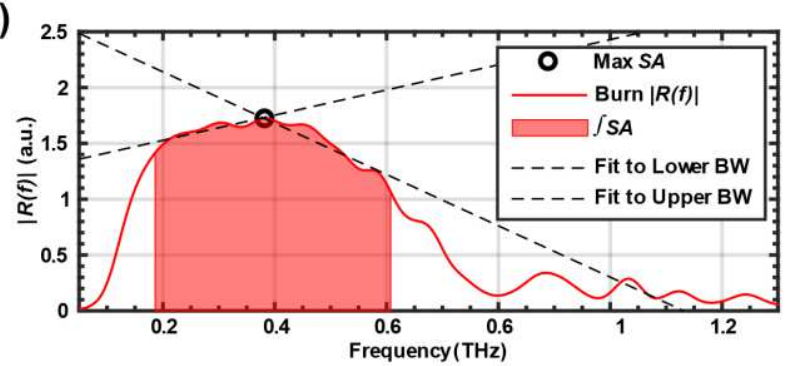

(b)

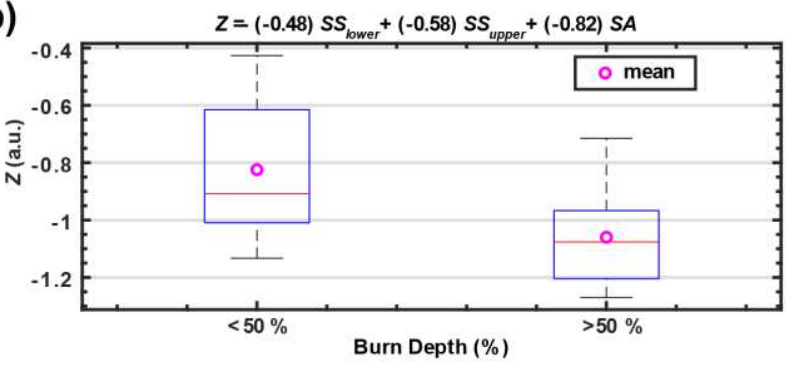


Fig. 2. THz Hyperspectral and statistical analysis. a) The spectral slopes of $R(f)$ when calculated using the upper and lower BW. b) A boxplot for the $Z$ parameter when compared to histological burn depth assessment is shown for burn depths greater than and less than 50\%. 1-way ANOVA showed statistical significance $(p=0.0016)$.

To utilize both the $S A$ and dual-band $S S$, we optimized a $Z$ parameter, defined as,

$$
Z=a * S S_{\text {lower }}+b * S S_{\text {upper }}+c * S A,
$$

where $a, b$, and $c$ are weighting coefficients for each spectral feature and were optimized between -1 and 1 to equal $-0.48,0.58$, and 0.82 , respectively. Shown in the boxplot from Fig. 2(b), a oneway ANOVA showed statistical significance $(p=0.0016)$ for distinguishing burns greater than and less than 50\%. Spectral measurements contained in the ROI of all THz-TDSI images were included in this analysis.

When adnexal structures are destroyed in a full-thickness wound, reepithelialization must occur from wound margins in full thickness wounds ${ }^{27}$. In addition to the wound margin, new epithelium in partial thickness wounds originate from hair follicles and apocrine glands in pigs

and from pilosebaceous units and apocrine sweat glands in humans ${ }^{27,28}$. Considering the major role that adnexal structures play in wound healing, utilizing them as a contrast mechanism in the SS parameter in THz-TDSI for non-invasive imaging and monitoring provides a valuable window to monitor burn wound healing, care, assessment and coverage.

\section{Wound progression}

In addition to differentiating burns greater than and less than 50\%, we used the THz-TDSI scanner to monitor the wound progression over the course of the study. We used the same $a, b$ and $c$ coefficients optimized for the hyperspectral Z parameter on Day 4 to calculate the Z-value for $\mathrm{THz}$ measurements obtained on Days 0 to 3. Fig. 3(a, c, e ,g) shows the locations where we marked ROI's on each day. To ensure an unbiased comparison between days and account for ROI location 
differences, we created 5 randomized subsets of 8 pixels within each ROI and show the standard deviation as the error bar in Fig. 3(b, d, f, h).

(a)
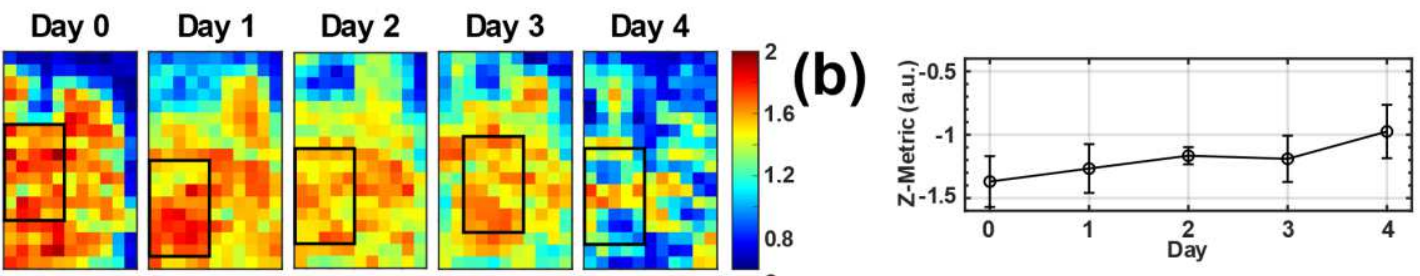

(c)
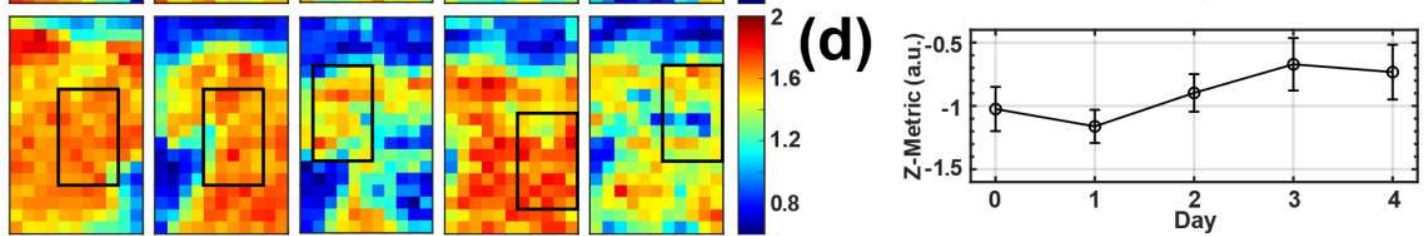

(e)
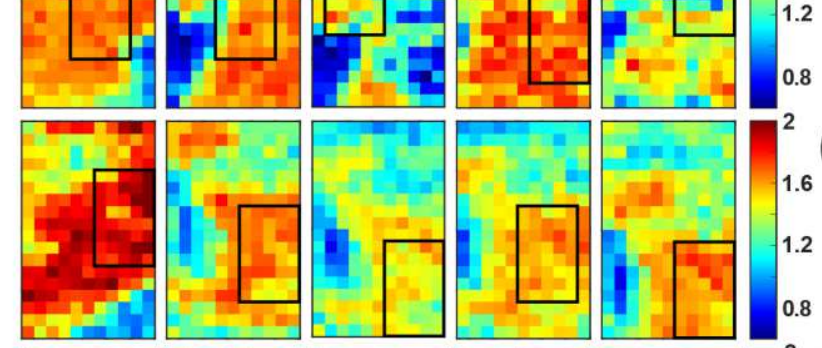

(g)
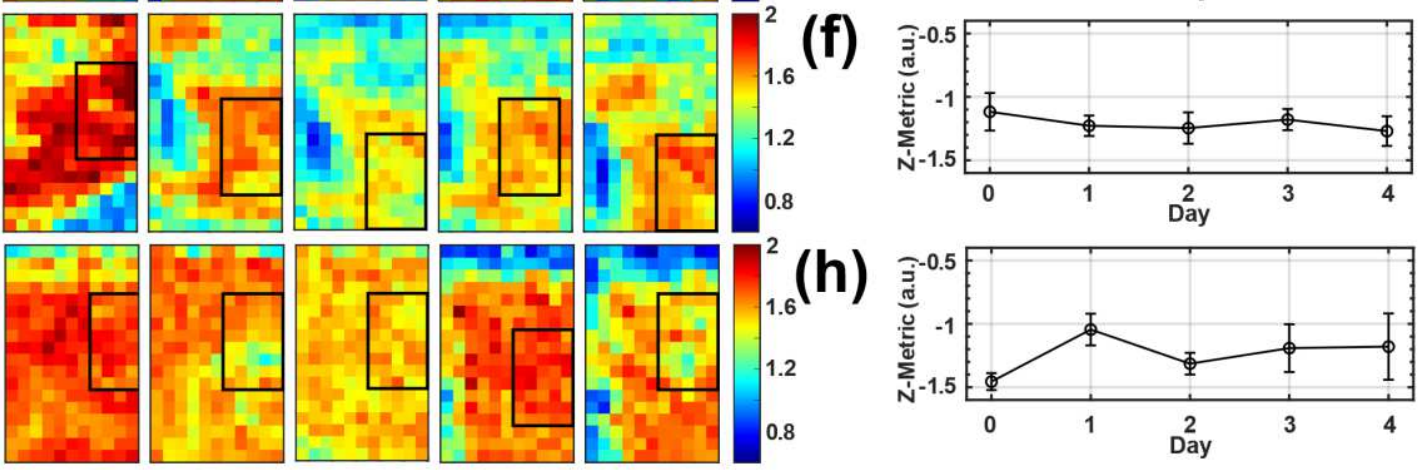

(i)
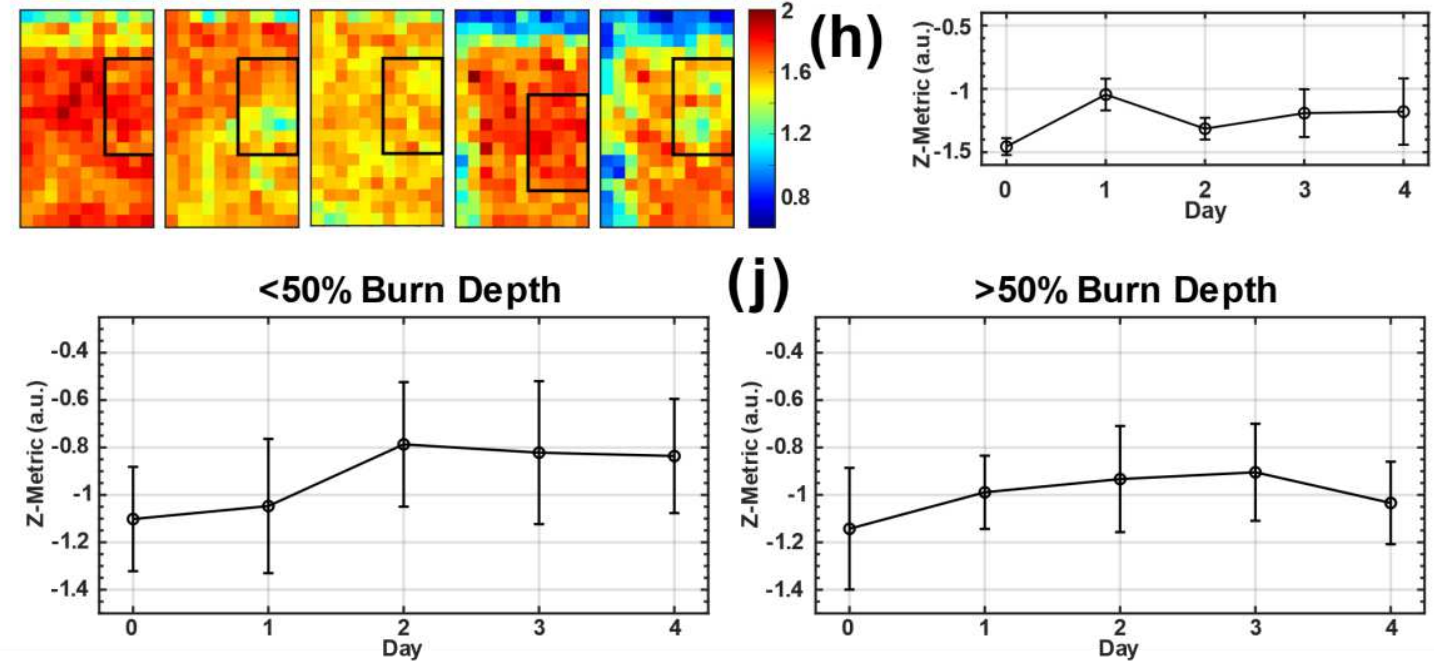

\section{(j)}

$>50 \%$ Burn Depth

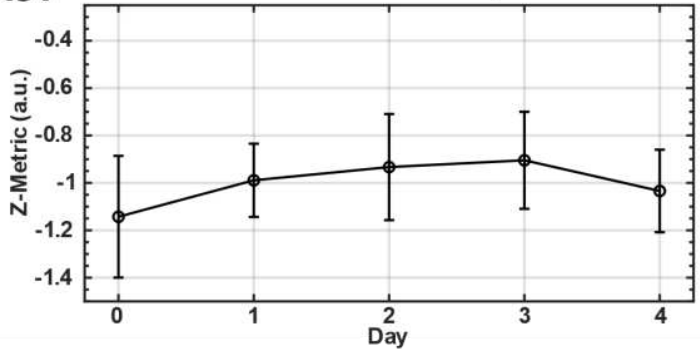

Fig. 3. Monitoring wound healing with THz-TDSI. The daily normalized integral of the spectral amplitude, $S A$, ROI locations (pixel size is $1 \times 1 \mathrm{~mm}^{2}$ ) and $Z$-parameter values (mean+/- SD) are shown for a representative superficial partial thickness injury with $22 \%$ burn depth (a-b), superficial partial thickness injury with 37\% burn depth (c-d), deep-partial thickness injury with 65\% burn depth (e-f), and full thickness injury with $100 \%$ burn depth (g-h). When considering all burns in this study, $Z$ parameter increased over time in burns less than 50\% depth (i) but remained relatively constant for burns greater than 50\% depth (j). 
Fig. 3 (a-d) shows a marked increase in $Z$ for two representative superficial partial-thickness burns whose depths were $22 \%$ and $37 \%$, respectively. When considering all burns in the study which have less than 50\% depth, Fig. 3 (i) shows that $Z$ also had an increasing trend over the course of the study. Fig. 3 (e-h) shows that $Z$ remained relatively constant in a representative deep partialthickness burn with 65\% depth and a full-thickness burn with 100\% depth. Similarly, Fig. 3 (j) shows that $Z$ remained constant when considering all burns over 50\%. A similar phenomenon is observed in laser doppler imaging, where perfusion increases in superficial burns but remains limited in deeper burns ${ }^{29}$. Compromised perfusion is a result of destroyed and damaged microvasculature post-burn and is an important factor for wound healing.

In this work, we used a handheld THz-TDSI scanner to characterize burn injuries in an acute in vivo porcine scald model. The scanner was designed and built by our research team and included a custom-fabricated $\mathrm{f}-\theta$ lens for $2 \mathrm{D}$ hyperspectral $\mathrm{THz}$ imaging. We showed that, by using the deconvolved spectral amplitude, we could demarcate burned and healthy tissue zones. Including the dual-band spectral slope in the hyperspectral Z-parameter allowed us to differentiate scald burns with depths greater or less than $50 \%$ as determined by histological assessment and monitor them over time. Our results suggest that the scattering loss from a THz-TDSI signal can be used to differentiate burn severities and monitor wound healing in partial thickness burns. Further in vivo studies will include improved image co-registration and handheld $\mathrm{THz}$ instrumentation hardware which has been scaled up to create a larger FOV, scan rate, and enhanced image processing algorithms.

\section{MATERIALS \& METHODS}

\section{$\underline{\text { Study protocol }}$}


The experimental protocol used in this study was reviewed and approved by the Institutional Animal Care and Use Committee (IACUC) at Stony Brook University. This study was carried out in compliance with the ARRIVE guidelines and all experiments were conducted in accordance with the protocol approved by IACUC at the Stony Brook University. The animal model was based on 12-weeks of age, female Landrace pigs, weighing approximately $25-30 \mathrm{~kg}$. Female pigs of this size were chosen based on previous works, which demonstrated reproducible animal burn models 30,31. Prior to inducing the burns on Day 0, the animal was sedated through an intramuscular (IM) injection of a pre-anesthetic cocktail consisting of ketamine $(20 \mathrm{mg} / \mathrm{kg})$, xylazine $(2.2 \mathrm{mg} / \mathrm{kg})$, acepromazine $(0.1 \mathrm{mg} / \mathrm{kg})$, and atropine $(0.02 \mathrm{mg} / \mathrm{kg})$, and then anesthetized with a continuous flow of $0.5-5 \%$ isoflurane. The pig skin was washed and the hair on its back was removed by trimming and shaving. A stencil was used to mark the locations for burn induction and guide the tattoo margins for tissue identification and image co-registration. Following burn induction, buprenorphine $(0.005-0.02 \mathrm{mg} / \mathrm{kg})$ was administered IM and a 72-hour transdermal fentanyl patch $(50 \mu \mathrm{g} / \mathrm{kg})$ was placed proximal to the tail. Additionally, tattoos were placed to note the tissue column and row identification and the location of each burn. These corner tattoos acted as guides for the placement of the handheld scanner and ensured that the device was placed at the same general area every day during the imaging studies. Following the tattoo procedure, the burns were debrided by gently scraping the burn with the blunt end of forceps.

The animal was kept on isoflurane throughout the imaging process and monitored by the veterinary staff of the Division of Laboratory Animal Research at Stony Brook University. After imaging, the burns were bandaged by applying triple antibiotic ointment to the individual injuries and covering the wounds with a transparent Tegaderm ${ }^{\mathrm{TM}}$ sheet (3M $\mathrm{M}^{\mathrm{TM}}$, Saint Paul, MN, USA). The midsection of the pig was then wrapped with flexible gauze bandage and adhesive Tensoplast $®$ 
(BSN Medical, Hamburg, Germany). On Days 1 to 4, prior to imaging, the animal was sedated and anesthetized with the same procedure as Day 0. After imaging on Day 4, the entire burn and perilesional tissue was collected for histological assessment and the animal was euthanized through intravenous administration of Fatal Plus $(100 \mathrm{mg} / \mathrm{kg})$.

Scald burns were created on the dorsal side of the pig using the device described in Fig. 4(a), where a foam wrapped steel pipe contained a hot water inflow tube and a suction tube outlet ${ }^{31}$. Water was kept at $97^{\circ} \mathrm{C}$ using an immersion heating circulator and flowed into the device through high-temperature rubber tubing using the circulation feature on the immersion heating device. Water temperature dropped by approximately $2^{\circ} \mathrm{C}$ as it flowed through the tubing for a final temperature of $95^{\circ} \mathrm{C}$ at the surface of the skin. Hot water was removed from the device using a vacuum pump (Adafruit Industries, New York, NY, USA) attached to a high-density polyethylene (HDPE) vacuum flask. Varied burn severities were created by exposure to $95^{\circ} \mathrm{C}$ water for 5,15 , and 25 seconds, using a $20 \times 20 \mathrm{~mm}^{2}$ square shaped scald device pipe. 

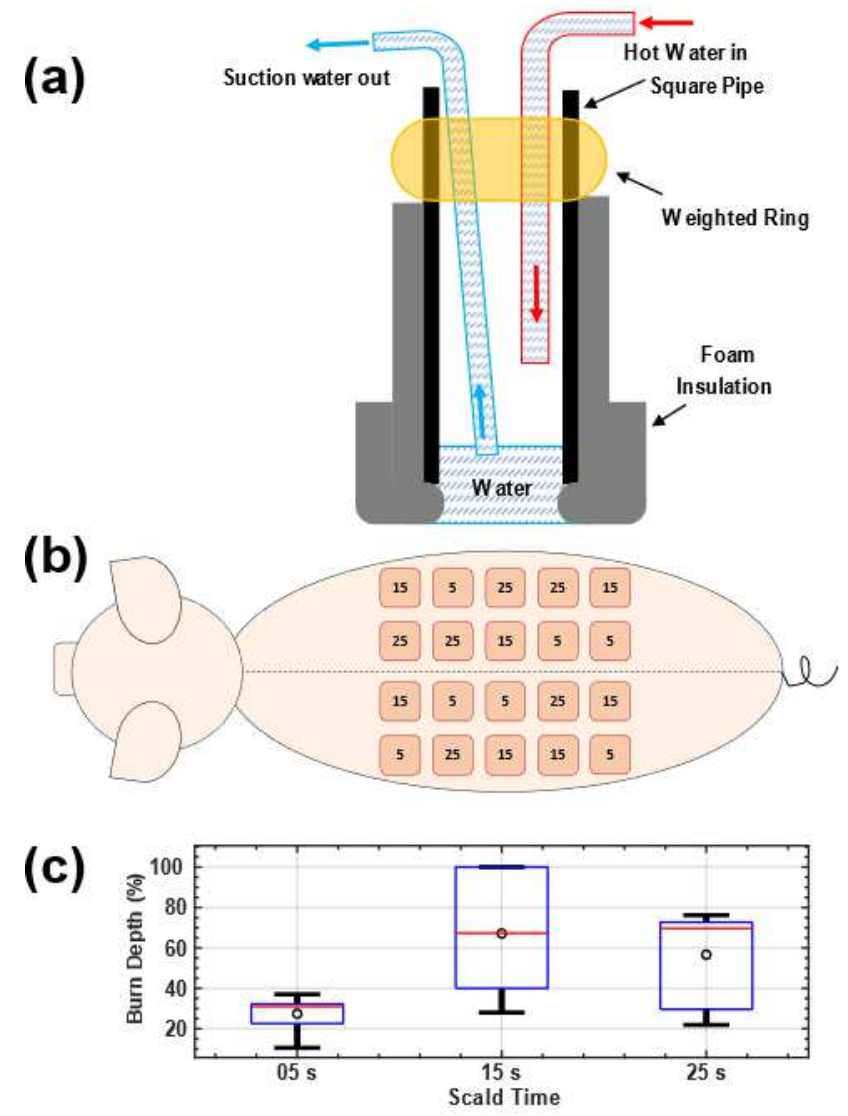

Fig. 4. Animal model. (a) The schematic of the scald burn induction device is shown, where $95^{\circ} \mathrm{C}$ water would come in direct contact with the skin. (b) Scald condition locations are illustrated where the numbers in each circle represent the heat exposure duration in seconds. (c) Dermal burn depth percentage (normalized by total dermal thickness) are plotted for each experimental condition, as determined by a histopathologist using Day-4 H\&E sections.

Burns were systematically distributed $(\mathrm{n}=20)$ to minimize variation due to the dorsal location. As illustrated in Fig. 4(b), an equal number of burns in each experimental arm are created in cranial and caudal sections, in proximal and distal locations to the spine. Based on the size of the animal and previous scald models ${ }^{31}$, the 5 -second exposure condition was selected to create a superficial burn, whereas the 25 -second condition was intended to create a full-thickness burn. The 15 -second condition was selected to create partial-thickness burns that would either progress into a fullthickness burn or continue the healing process by Day 4 as a superficial partial-thickness injury. 
This dynamic progression effect in the 15-second burns can be seen in Fig. 4(c) by the large variance in the burn depth compared to the 5- and 25-second conditions.

\section{$\underline{\text { Histology }}$}

At the conclusion of the study, a $50 \times 50 \mathrm{~mm}^{2}$ tissue block that included perilesional and burned skin was excised and fixed in formalin for 24 hours before storage in alcohol. Punch biopsies (4$\mathrm{mm}$ diameter) were used to obtain tissue samples from various locations and stained with hematoxylin and eosin (H\&E). Histological examples of each scald condition are shown in Fig. 5. Blind evaluation was performed by a board-certified histopathologist. The burn depth was assessed by measuring the deepest point of injury, characterized by microvascular occlusion, collagen discoloration, follicular cell necrosis, mesenchymal cell necrosis, and adipocyte necrosis for very deep burns ${ }^{32}$. The injury depth was then divided by the total dermal thickness and multiplied by 100 to calculate the percent burn depth.
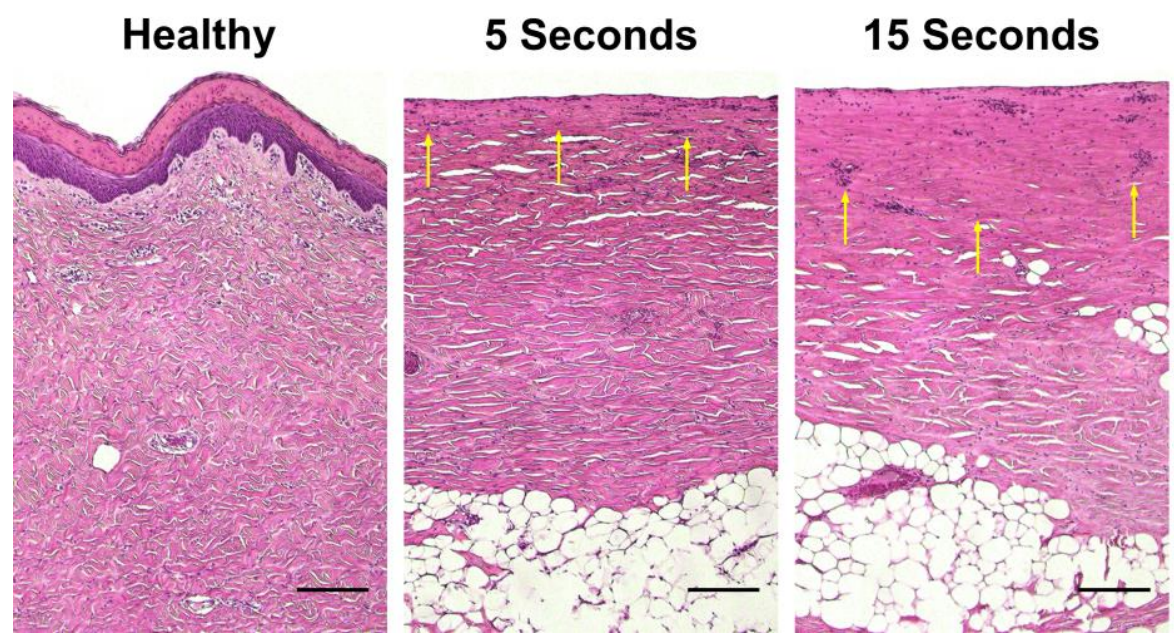

25 Seconds

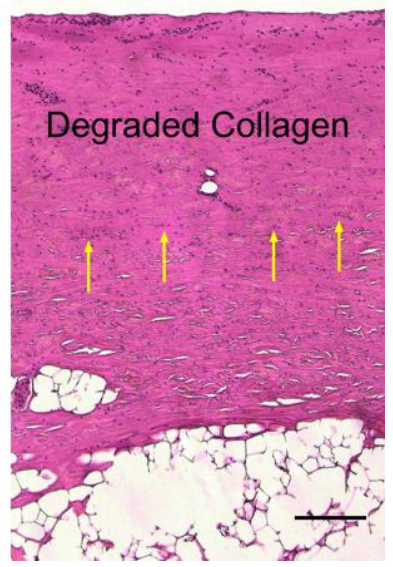

Fig. 5. Histology results. Images of H\&E stained skin samples are shown for each scald condition. Images were captured using a Nikon mm-60 microscope (Nikon Instruments Inc. Tokyo, Japan) with a 5x objective lens. Yellow arrows indicate pyknotic cells (scale bar $=0.2 \mathrm{~mm}$ ).

\section{Handheld THz-TDSI scanner}


$\mathrm{THz}$ radiation was generated and detected using photoconductive antennas (PCA) as part of an Asynchronous Optical Sampling (ASOPS, Menlo Systems Inc, Newton, NJ) THz-TDS system. The ASOPS THz-TDS system uses two $1560 \mathrm{~nm}$ femtosecond lasers, one used to pump an InGaAs/InAlAs THz PCA emitter and the other to probe a LT InGaAs/InAlAs THz PCA detector. Each laser is set to a slightly offset repetition rate to sample the probe pulse in the time-domain. This is beneficial because, rather than sampling the TD signal in the conventional method that utilizes a motorized stage delay line, we sample the TD signal electronically for much faster data acquisition time while maintaining high signal to noise. The laser repetition rate was set to 100 $\mathrm{MHz}$, with the sampling rate set by the ASOPS difference frequency equal to $100 \mathrm{~Hz}$, which resulted in measurement of a full $\mathrm{THz}$ time-domain signal every 0.01 seconds.

THz scans were performed using a Portable HAndheld Spectral Reflection (PHASR) scanner 24,33. The design of the PHASR scanner, shown in Fig. 6(a), features a custom HDPE f- $\theta$ lens and a mirror mounted on a motorized 2-axis gimbal stage (T-OMG, Zaber Technologies Inc. Vancouver, BC, Canada) in a telecentric configuration ${ }^{32,33}$. The $12 \times 19 \mathrm{~mm}^{2}$ area can be scanned in approximately two minutes with a depth of focus much greater than the penetration depth of the $\mathrm{THz}$ beam ${ }^{25}$. Furthermore, the scanner is "alignment-free" and maintains constant resolution across the entire FOV. Fig.6(b) shows the handheld THz scanner in an operating theatre to image porcine scald burns created in this study. The design of the PHASR scanner was pivotal for this in vivo porcine study because large animals cannot easily be raster scanned. Additionally, their physiological movements like respiration would cause significant imaging artifacts and potential misalignment. Using the PHASR instrument allowed for the movement of the scanning optics in tandem with any animal movements, similar to a portable ultrasound scanner, and therefore enabled an alignment-free and fast imaging operation. 


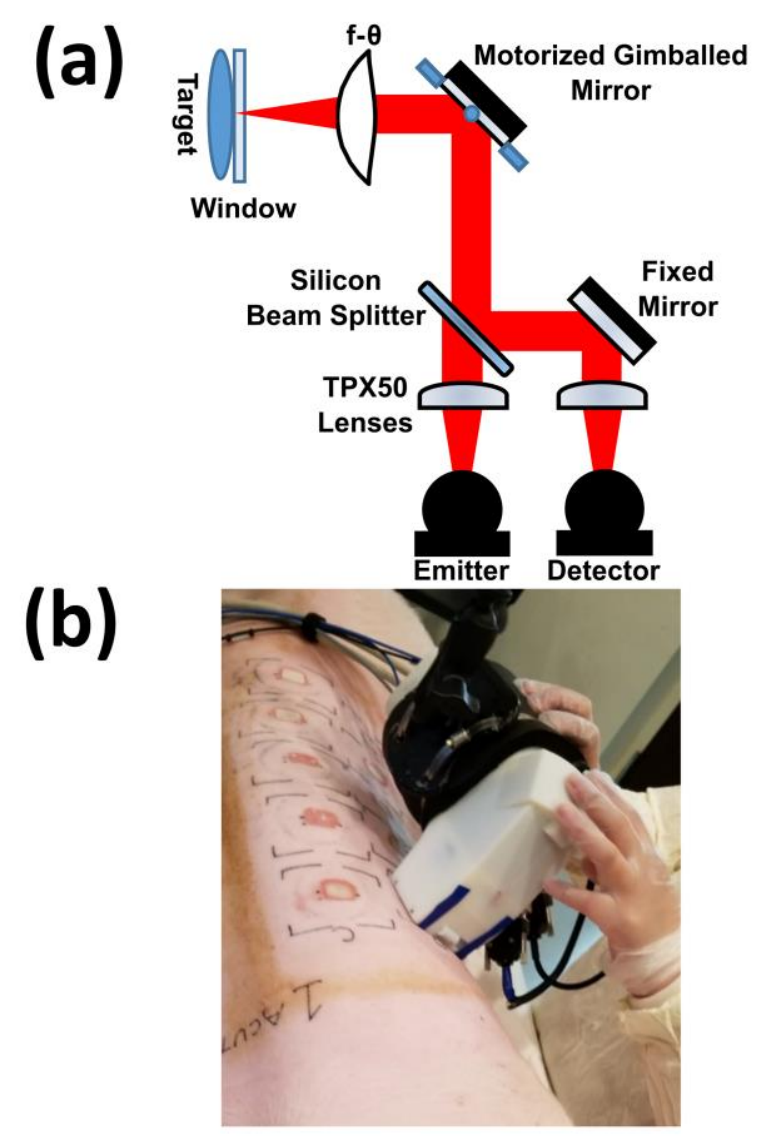

Fig. 6. In vivo handheld THz setup. (a) The optical schematic for the handheld THz scanning system. (b) A visual image of the handheld scanner in use.

Further details about the $\mathrm{THz}$ handheld scanner can be found in Table 1. When compared to alternative advanced optical imaging techniques such as optical coherence tomography or photoacoustic microscopy, THz systems can increase the FOV by scaling up the hardware while maintaining sub-second pixel scan times. Additionally, THz-TDSI directly measures the optical properties of the wound, while techniques such as laser doppler imaging and thermography rely exclusively on physiological phenomenon, i.e. perfusion, as a contrast mechanism.

\begin{tabular}{|c|c|c|c|c|c|}
\hline $\begin{array}{c}\text { Spatial } \\
\text { Resolution (mm) }\end{array}$ & $\begin{array}{c}\text { Depth of Focus } \\
(\mathbf{m m})\end{array}$ & FOV $\left(\mathbf{m m}^{2}\right)$ & $\begin{array}{c}\text { Scan Time } \\
(\mathbf{s e c o n d s})\end{array}$ & $\begin{array}{c}\text { Pixel Size } \\
(\mathbf{m m})\end{array}$ & $\begin{array}{c}\text { THz Intensity } \\
\left(\mathbf{m W} / \mathbf{c m}^{\mathbf{2}}\right)\end{array}$ \\
\hline 0.76 & 9.55 & up to $43 \times 27$ & $\geq 0.25$ per pixel & 1 & Approx. $<0.37$ \\
\hline
\end{tabular}

Table 1. Handheld scanner specifications: Specifications for the handheld THz-TDSI scanner developed for burn diagnostic imaging. 


\section{$\underline{\text { Signal processing }}$}

The complete signal processing steps are outlined by the flowchart in Fig. 7. All analysis was performed using the MATLAB software (MathWorks Inc, Natick, MA, USA). The raw TD signal in each pixel was first baseline corrected ${ }^{35}$ to remove any inherent signal artifacts. A Gaussian bandpass filter, containing signal in our usable BW (0.05 to $2.5 \mathrm{THz}$ pass band), along with a zeropadded split-Blackman window were applied to the TD signal before applying a Fourier Transform. The complex Fourier-domain representation of the signal, defined as $E_{\text {samp }}(f)$, was deconvolved by a reference signal, $E_{r e f}(f)$, with the same signal processing steps as the sample. To minimize the impact of noise where the signal-to-noise ratio of the spectra is poor, a Wiener deconvolution algorithm was implemented, where the deconvolved spectral amplitude, $R(f)$, is given by 36

$$
R(f)=E_{\text {samp }}(f) \frac{E_{r e f}^{*}(f)}{E_{r e f}(f) E_{r e f}^{*}(f)+\alpha<E>\left[E_{r e f}(f) E_{r e f}^{*}\right]}
$$

The Wiener deconvolution implementation uses a Tikhonov Regularization method ${ }^{37}$, where < $E>$ is the expected value and $\alpha$ is the regularization constant.

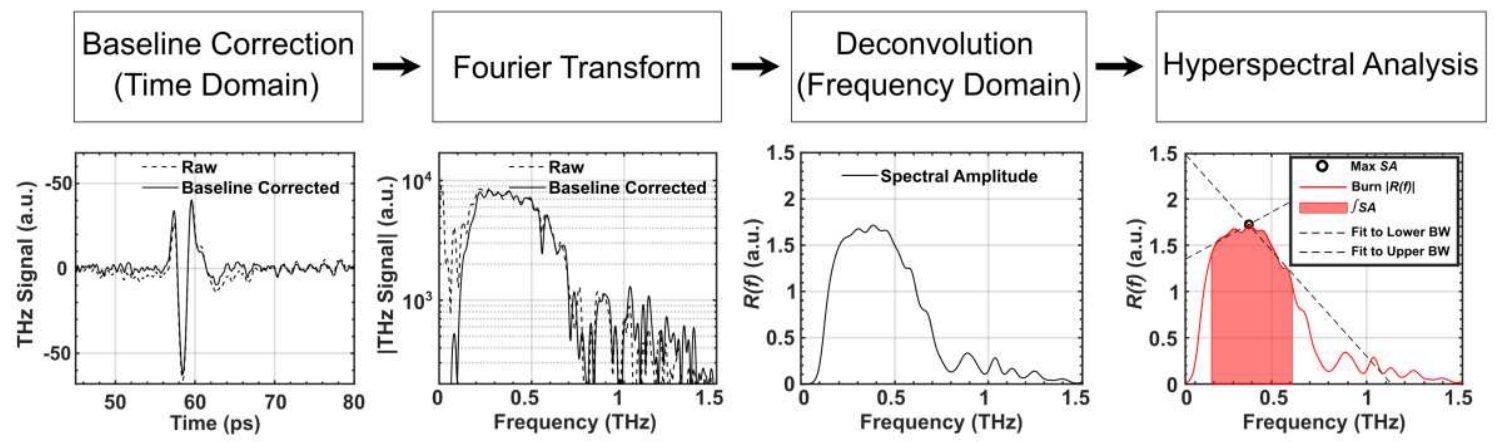

Fig. 7. THz signal processing steps. The signal processing flowchart is shown with selected steps depicted below and noted where analysis is performed in the time or frequency domain. 


\section{CONFLICTS OF INTEREST}

P: MHA discloses intellectual property owned by the University of Washington, US Patent No. US9295402B1.

\section{ACKNOWLEDGEMENTS}

The authors would like to acknowledge the Stony Brook University Division of Laboratory Animals and Resources for their support in the porcine study.

\section{FUNDING}

The National Institute of General Medical Sciences (R01GM112693).

\section{AUTHOR CONTRIBUTIONS}

Conceptualization: MHA; Data Curation: ZBH, OBO, JWZ; Formal Analysis: OBO; Funding Acquisition: MHA; Investigation: OBO, JWZ, ZBH; Methodology: MHA, AJS, JWZ; Project Administration: MHA, AJS; Resources: MHA; Software: ZBH, OBO, JWZ, MEK; Supervision: MHA, AJS; Validation: MHA, OBO, AJS; Visualization: OBO; Writing - OBO; Writing Review and Editing: OBO, MHA, JWZ, ZBH, MEK, AJS.

\section{DATA AVAILABILITY}

The datasets generated during and/or analyzed during the current study are available from the corresponding author on reasonable request.

\section{REFERENCES}

1. Rui, P. \& Kang, K. National Hospital Ambulatory Medical Care Survey: 2017 emergency department summary tables. (US Department of Health and Human Services, Centers for Disease Control, 2017).

2. Devgan, L., Bhat, S., Aylward, S. \& Spence, R. J. Modalities for the assessment of burn wound depth. J. Burns Wounds 5, (2006). 
3. Kim, D. E. et al. Microvascular Assessment of Burn Depth Conversion during Varying Resuscitation Conditions. J. Burn Care Rehabil. 22, 406-416 (2001).

4. Ward, P. A. \& Till, G. O. Pathophysiologic events related to thermal injury of skin. J. Trauma 30, S75-79 (1990).

5. Atiyeh, B. S., Gunn, S. W. \& Hayek, S. N. State of the art in burn treatment. World J. Surg. 29, 131148 (2005).

6. Ong, Y. S., Samuel, M. \& Song, C. Meta-analysis of early excision of burns. Burns 32, 145-150 (2006).

7. Singer, A. J. et al. Early versus Delayed Excision and Grafting of Full-Thickness Burns in a Porcine Model: A Randomized Study. Plast Reconstr Surg 137, 972e-9e (2016).

8. Heyden, M. et al. Dissecting the THz spectrum of liquid water from first principles via correlations in time and space. Proc. Natl. Acad. Sci. 107, 12068-12073 (2010).

9. Yang, X. et al. Biomedical applications of terahertz spectroscopy and imaging. Trends Biotechnol. 34, 810-824 (2016).

10. Siegel, P. H. Terahertz Technology in Biology and Medicine. IEEE Trans. Microw. Theory Tech. 52, $2438-2447$ (2004).

11. Arbab, M. H. et al. Terahertz reflectometry of burn wounds in a rat model. Biomed. Opt. Express 2 , 2339-2347 (2011).

12. Arbab, M. H. et al. Terahertz spectroscopy for the assessment of burn injuries in vivo. J. Biomed. Opt. 18, 077004 (2013).

13. Wilmink, G. J. \& Grundt, J. E. Invited review article: current state of research on biological effects of terahertz radiation. J. Infrared Millim. Terahertz Waves 32, 1074-1122 (2011).

14. ANSI. American National Standard for the Safe Use of Lasers. Z136.1 1, (2014).

15. Taylor, Z. D. et al. Reflective terahertz imaging of porcine skin burns. Opt. Lett. 33, 1258-1260 (2008). 
16. Tewari, P. et al. In vivo terahertz imaging of rat skin burns. J. Biomed. Opt. 17, 0405031-0405033 (2012).

17. Rowan, M. P. et al. Burn wound healing and treatment: review and advancements. Crit. Care 19, 243 (2015).

18. Arbab, M. H. et al. A Noninvasive Terahertz Assessment of 2nd and 3rd Degree Burn Wounds. in (Optical Society of America, 2012).doi:10.1364/CLEO_SI.2012.CTu3B.3.

19. Osman, O. B. et al. Differentiation of burn wounds in an in vivo porcine model using terahertz spectroscopy. Biomed. Opt. Express 11, 6528-6535 (2020).

20. Suen, J. Y. \& Padilla, W. J. Superiority of terahertz over infrared transmission through bandages and burn wound ointments. Appl. Phys. Lett. 108, 233701 (2016).

21. Zhang, X. \& Redo-Sanchez, A. Handheld THz Instrumentation. SPIE Prof. (2012).

22. Echchgadda, I. et al. Using a portable terahertz spectrometer to measure the optical properties of in vivo human skin. J. Biomed. Opt. 18, 120503 (2013).

23. Grootendorst, M. R. et al. Use of a handheld terahertz pulsed imaging device to differentiate benign and malignant breast tissue. Biomed. Opt. Express 8, 2932-2945 (2017).

24. Harris, Z. B., Khani, M. E. \& Arbab, M. H. Terahertz Portable Handheld Spectral Reflection (PHASR) Scanner. IEEE Access (2020).

25. Harris, Z., Virk, A., Khani, M. \& Arbab, M. H. Terahertz time-domain spectral imaging using telecentric beam steering and an $\mathrm{f}-\theta$ scanning lens: distortion compensation and determination of resolution limits. Opt. Express (2020) doi:10.1364/OE.398706.

26. Shen, Y. C., Taday, P. \& Pepper, M. Elimination of scattering effects in spectral measurement of granulated materials using terahertz pulsed spectroscopy. Appl. Phys. Lett. 92, 051103 (2008).

27. Rittié, L. Cellular mechanisms of skin repair in humans and other mammals. J. Cell Commun. Signal. 10, 103-120 (2016).

28. Li, J., Chen, J. \& Kirsner, R. Pathophysiology of acute wound healing. Clin. Dermatol. 25, 9-18 (2007). 
29. Droog, E. J., Steenbergen, W. \& Sjöberg, F. Measurement of depth of burns by laser Doppler perfusion imaging. Burns 27, 561-568 (2001).

30. Singer, A. J. et al. Validation of a vertical progression porcine burn model. J Burn Care Res 32, 638$46(2011)$.

31. Andrews, C. J., Kempf, M., Kimble, R. \& Cuttle, L. Development of a consistent and reproducible porcine scald burn model. PloS One 11, e0162888 (2016).

32. Singer, A. J., Berruti, L., Thode Jr, H. C. \& McClain, S. A. Standardized burn model using a multiparametric histologic analysis of burn depth. Acad. Emerg. Med. 7, 1-6 (2000).

33. Harris, Z. B., Virk, A. \& Arbab, M. H. Handheld Telecentric THz-TDS Scanner Using Custom f\$peta\$ Optics for Imaging Applications in Clinical Settings and Non-Destructive Testing. in 2019 44th International Conference on Infrared, Millimeter, and Terahertz Waves (IRMMW-THz) 1-1 (IEEE, 2019).

34. Harris, Z. B., Katletz, S., Khani, M. E., Virk, A. \& Arbab, M. H. Design and characterization of telecentric $\mathrm{f}-\theta$ scanning lenses for broadband terahertz frequency systems. AIP $A d v \mathbf{1 0}, 125313$ (2020).

35. Huang, S. Y. et al. Tissue characterization using terahertz pulsed imaging in reflection geometry. Phys. Med. Biol. 54, 149 (2009).

36. Chen, Y., Huang, S. \& Pickwell-MacPherson, E. Frequency-wavelet domain deconvolution for terahertz reflection imaging and spectroscopy. Opt. Express 18, 1177-1190 (2010).

37. Tikhonov, A. N. \& Arsenin, V. I. Solutions of ill-posed problems. vol. 14 (Winston, Washington, DC, 1977). 


\section{TABLES AND FIGURE LEGENDS}

Fig. 1: Results from THz imaging. Visual images (a-b), THz images (c-d), where the quantity shown in the colorbar is $S A$, and representative $\mathrm{THz}$ reflectivity of burned (red box) and normal (black box, control) skin within the defined ROI (e-f) (mean +/- SD) are shown for each burn condition on Day 0 and Day 4. There are two side-by-side THz images shown for each $20 \times 20 \mathrm{~mm}^{2}$ burn and the surrounding normal skin region, because the FOV of the THz scanner was limited to $12 \times 19 \mathrm{~mm}^{2}$. (Scale bar $\left.=5 \mathrm{~mm}\right)$.

Fig. 2: THz Hyperspectral and statistical analysis. a) The spectral slopes of $R(f)$ when calculated using the upper and lower BW. b) A boxplot for the $Z$ parameter when compared to histological burn depth assessment is shown for burn depths greater than and less than 50\%. 1-way ANOVA showed statistical significance $(p=0.0016)$.

Fig. 3 Monitoring wound healing with THz-TDSI. The daily normalized integral of the spectral amplitude, $S A$, ROI locations (pixel size is $1 \times 1 \mathrm{~mm}^{2}$ ) and $Z$-parameter values (mean+/- SD) are shown for a representative superficial partial thickness injury with $22 \%$ burn depth (a-b), superficial partial thickness injury with $37 \%$ burn depth (c-d), deep-partial thickness injury with $65 \%$ burn depth (e-f), and full thickness injury with $100 \%$ burn depth (g-h). When considering all burns in this study, $Z$ parameter increased over time in burns less than $50 \%$ depth (i) but remained relatively constant for burns greater than $50 \%$ depth (j).

Fig. 4: Animal model. (a) The schematic of the scald burn induction device is shown, where $95^{\circ}$ $\mathrm{C}$ water would come in direct contact with the skin. (b) Scald condition locations are illustrated where the numbers in each circle represent the heat exposure duration in seconds. (c) Dermal burn depth percentage (normalized by total dermal thickness) are plotted for each experimental condition, as determined by a histopathologist using Day-4 H\&E sections.

Fig. 5: Histology results. Images of $H \& E$ stained skin samples are shown for each scald condition. Images were captured using a Nikon mm-60 microscope (Nikon Instruments Inc. Tokyo, Japan) with a 5x objective lens. Yellow arrows indicate pyknotic cells (scale bar $=0.2$ $\mathrm{mm})$.

Fig. 6. In vivo handheld THz setup (a) The optical schematic for the handheld $\mathrm{THz}$ scanning system. (b) A visual image of the handheld scanner in use.

Fig. 7 THz signal processing steps. The signal processing flowchart is shown with selected steps depicted below and noted where analysis is performed in the time or frequency domain.

Table 1: Handheld scanner specifications. Specifications for the handheld THz-TDSI scanner developed for burn diagnostic imaging.

\begin{tabular}{|c|c|c|c|c|c|}
\hline $\begin{array}{c}\text { Spatial } \\
\text { Resolution }(\mathrm{mm})\end{array}$ & $\begin{array}{c}\text { Depth of Focus } \\
(\mathrm{mm})\end{array}$ & FOV $\left(\mathrm{mm}^{2}\right)$ & $\begin{array}{c}\text { Scan Time } \\
(\text { seconds })\end{array}$ & $\begin{array}{c}\text { Pixel Size } \\
(\mathbf{m m})\end{array}$ & $\begin{array}{c}\text { THz Intensity } \\
\left(\mathrm{mW} / \mathrm{cm}^{2}\right)\end{array}$ \\
\hline
\end{tabular}




\begin{tabular}{|l|l|l|l|l|l|}
\hline 0.76 & 9.55 & up to $43 \times 27$ & $\geq 0.25$ per pixel & 1 & Approx. $<0.37$ \\
\hline
\end{tabular}




\section{Figures}

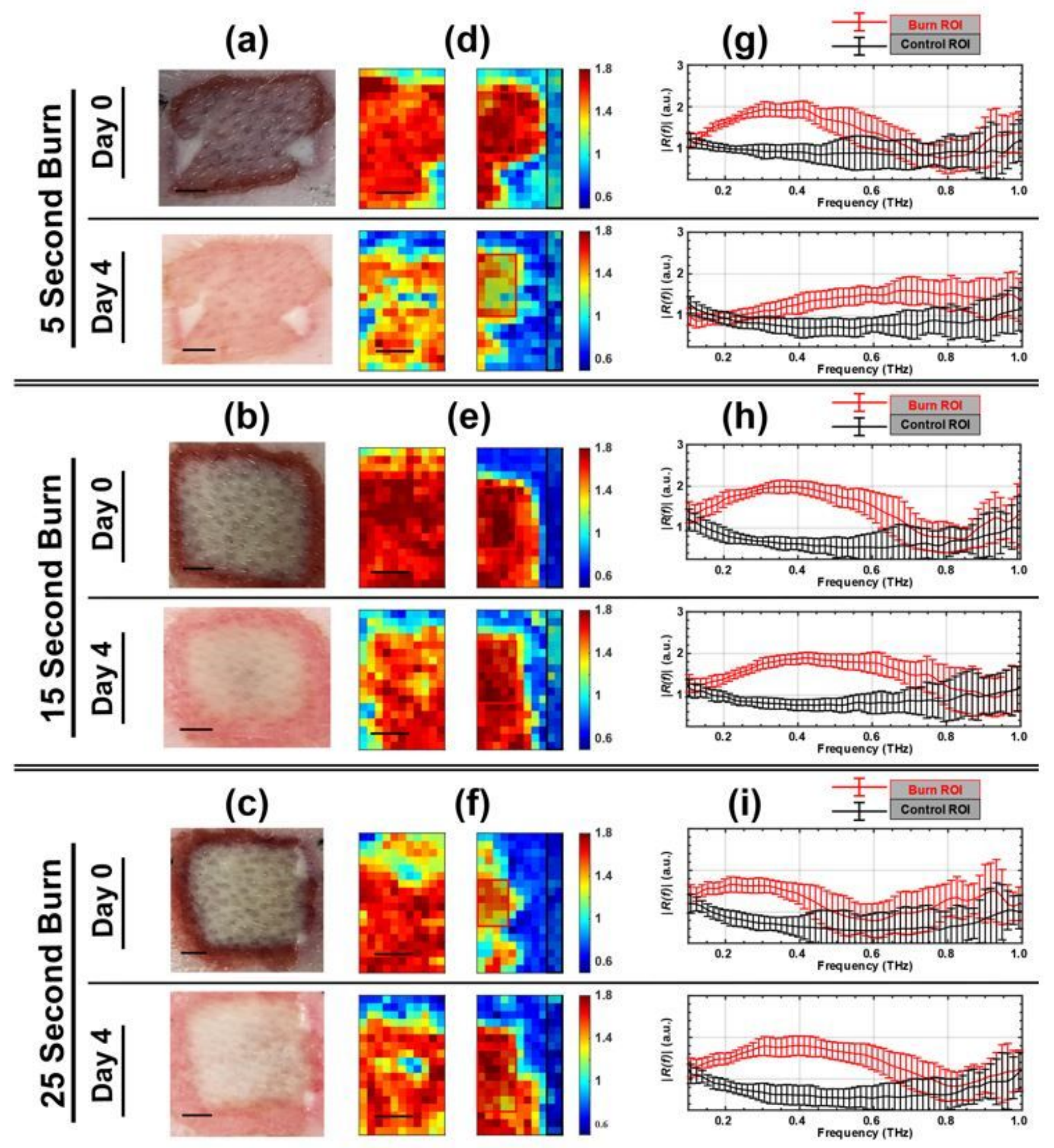

Figure 1

Results from THz imaging. Visual images (a-b), THz images (c-d), where the quantity shown in the colorbar is SA, and representative THz reflectivity of burned (red box) and normal (black box, control) skin within the defined ROI (e-f) (mean +/-SD) are shown for each burn condition on Day 0 and Day 4 . There 
are two side-by-side THz images shown for each $20 \times 20 \mathrm{~mm} 2$ burn and the surrounding normal skin region, because the FOV of the THz scanner was limited to $12 \times 19 \mathrm{~mm} 2$. (Scale bar $=5 \mathrm{~mm}$ ).
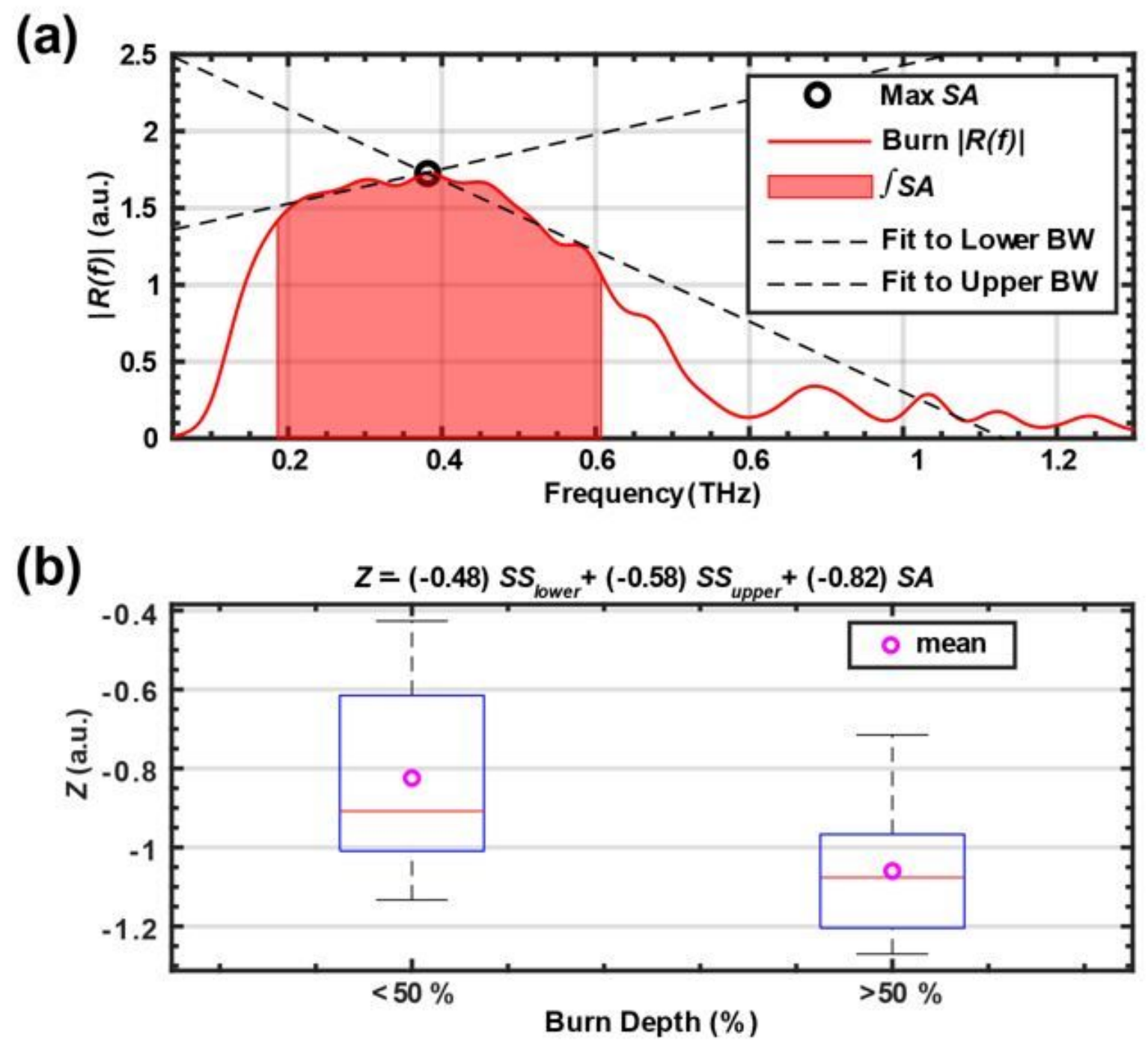

Figure 2

THz Hyperspectral and statistical analysis. a) The spectral slopes of $\mathrm{R}(\mathrm{f})$ when calculated using the upper and lower BW. b) A boxplot for the $Z$ parameter when compared to histological burn depth assessment is shown for burn depths greater than and less than 50\%. 1-way ANOVA showed statistical significance $(p=0.0016)$. 


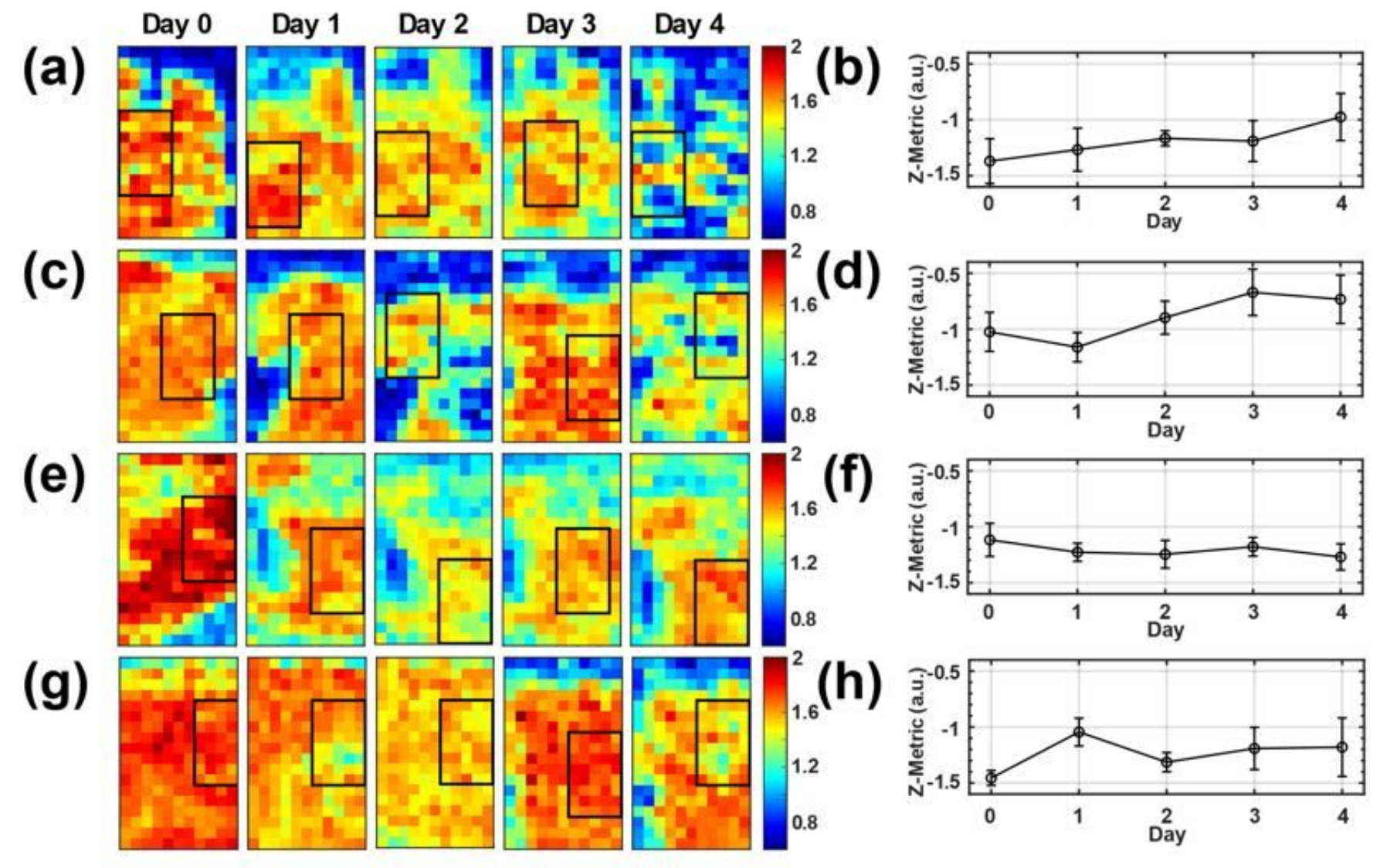

(i)
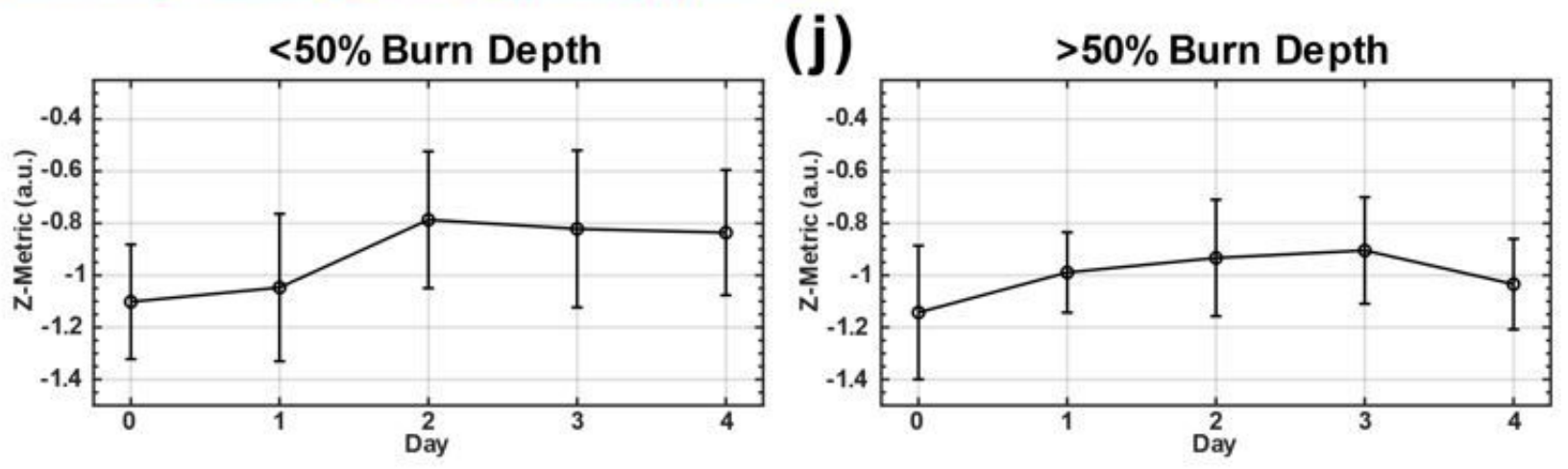

Figure 3

Monitoring wound healing with THz-TDSI. The daily normalized integral of the spectral amplitude, SA, ROI locations (pixel size is $1 \times 1 \mathrm{~mm} 2$ ) and Z-parameter values (mean+/-SD) are shown for a representative superficial partial thickness injury with $22 \%$ burn depth (a-b), superficial partial thickness injury with $37 \%$ burn depth (c-d), deep-partial thickness injury with $65 \%$ burn depth (e-f), and full thickness injury with $100 \%$ burn depth $(\mathrm{g}-\mathrm{h})$. When considering all burns in this study, Z parameter increased over time in burns less than $50 \%$ depth (i) but remained relatively constant for burns greater than $50 \%$ depth (j). 

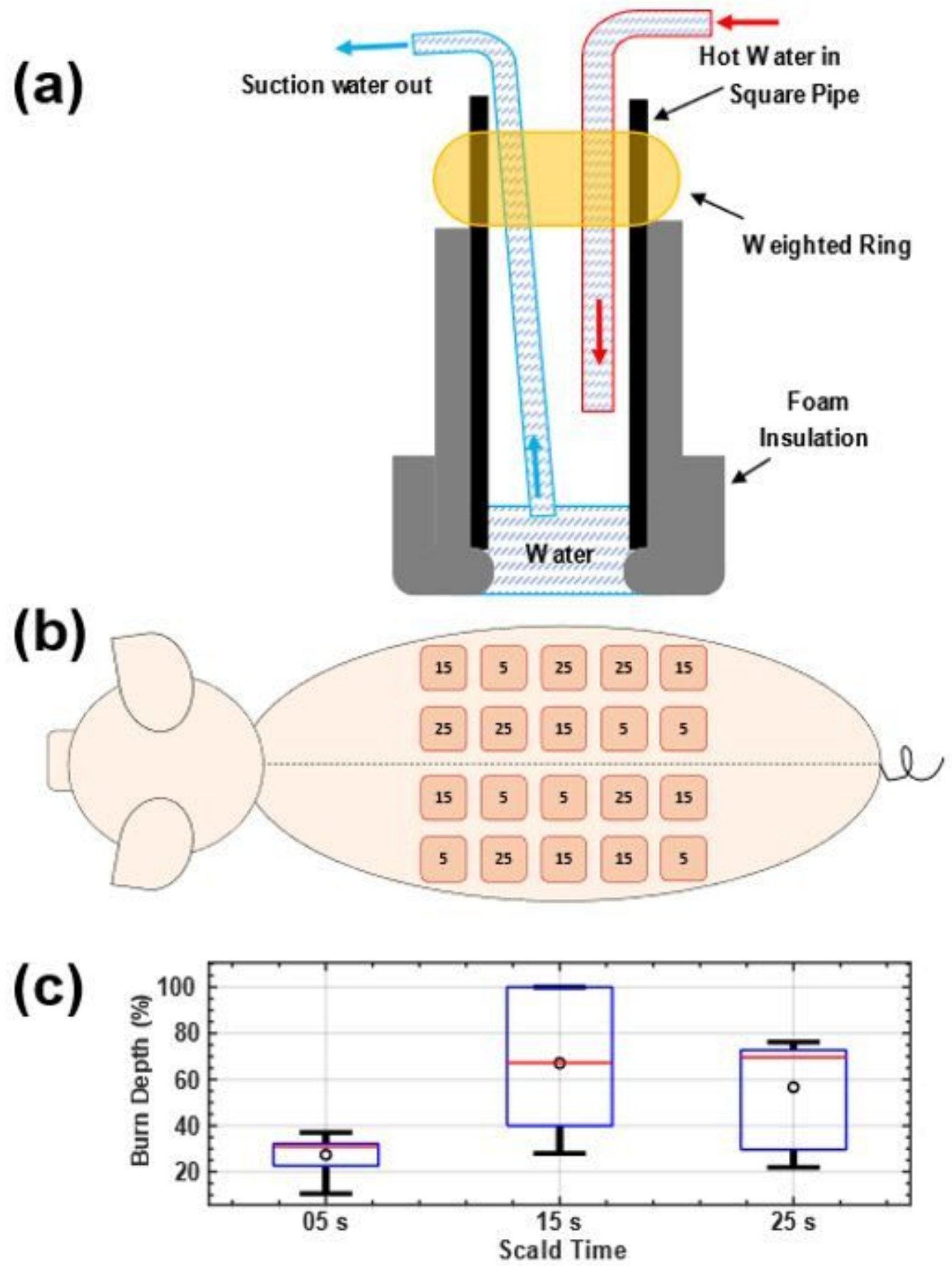

Figure 4

Animal model. (a) The schematic of the scald burn induction device is shown, where $95^{\circ} \mathrm{C}$ water would come in direct contact with the skin. (b) Scald condition locations are illustrated where the numbers in each circle represent the heat exposure duration in seconds. (c) Dermal burn depth percentage (normalized by total dermal thickness) are plotted for each experimental condition, as determined by a histopathologist using Day-4 H\&E sections. 


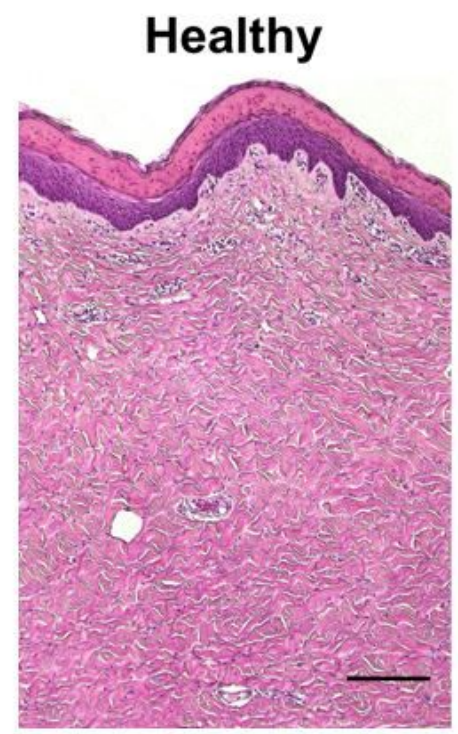

5 Seconds

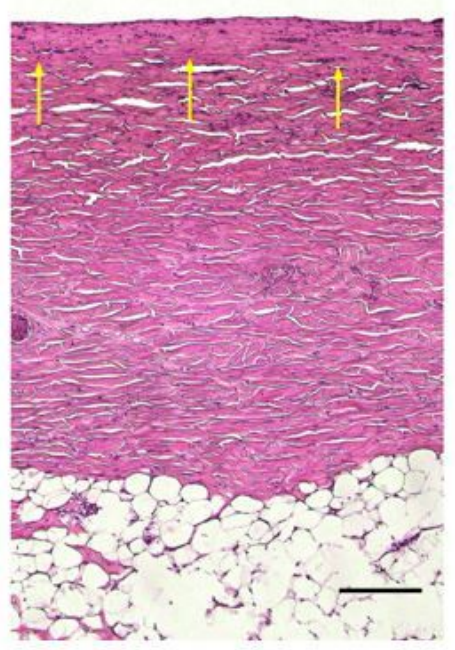

15 Seconds

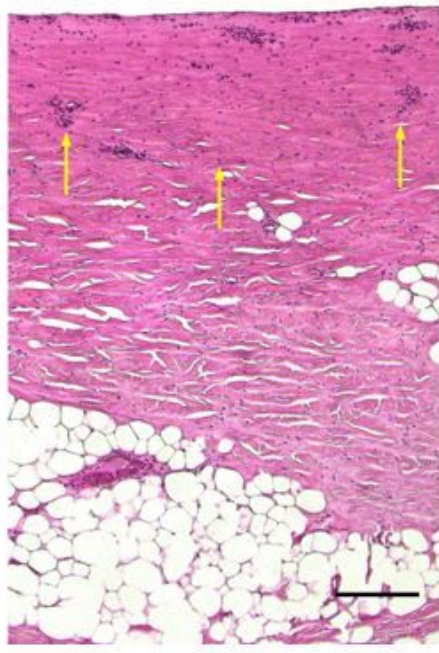

25 Seconds

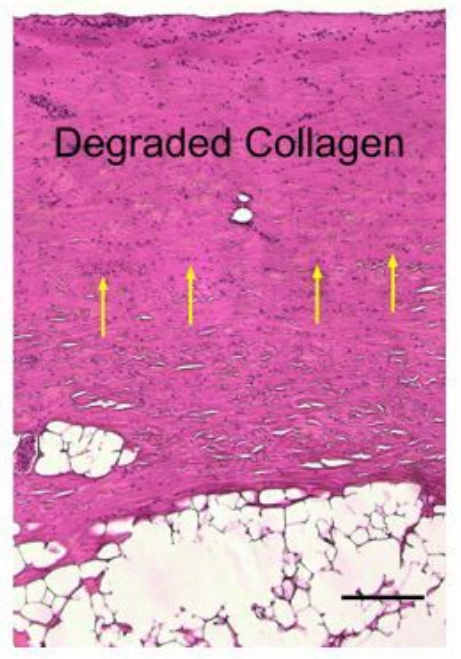

\section{Figure 5}

Histology results. Images of H\&E stained skin samples are shown for each scald condition. Images were captured using a Nikon mm-60 microscope (Nikon Instruments Inc. Tokyo, Japan) with a 5x objective lens. Yellow arrows indicate pyknotic cells (scale bar $=0.2 \mathrm{~mm}$ ). 


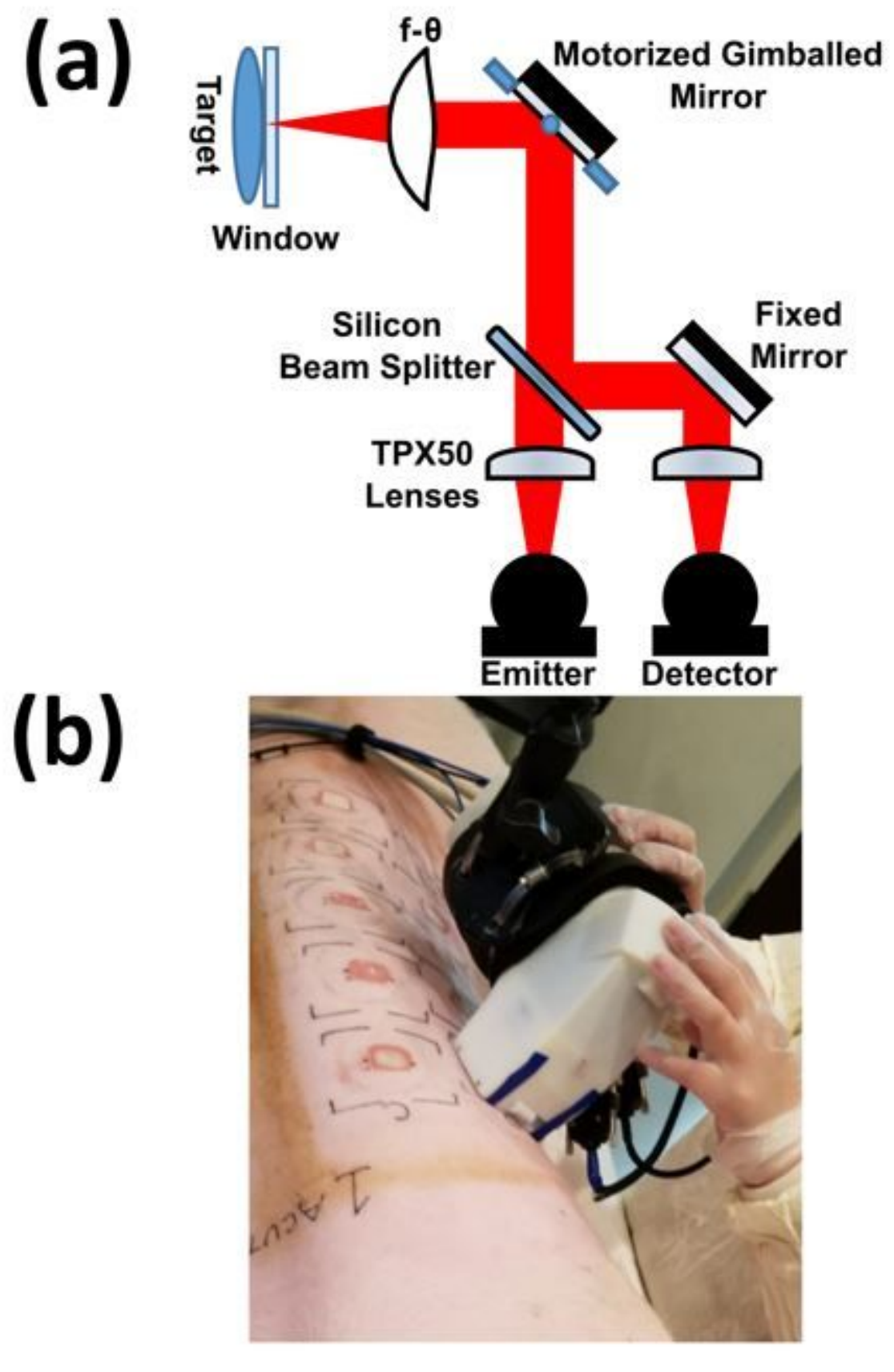

Figure 6

In vivo handheld $\mathrm{THz}$ setup (a) The optical schematic for the handheld $\mathrm{THz}$ scanning system. (b) A visual image of the handheld scanner in use. 


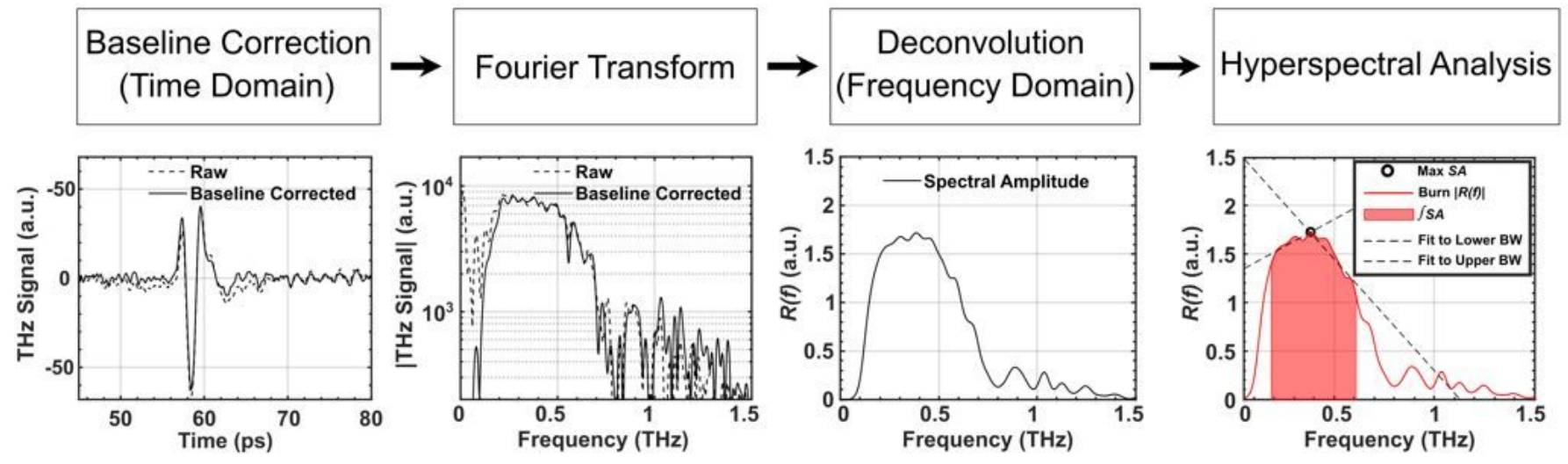

\section{Figure 7}

THz signal processing steps. The signal processing flowchart is shown with selected steps depicted below and noted where analysis is performed in the time or frequency domain. 